\title{
DRY DEPOSITION MODELLING IN A LAGRANGIAN DISPERSION MODEL
}

\author{
Helen $N$ Webster and David J Thomson
}

Met Office, Exeter, United Kingdom

\begin{abstract}
Revisions to the dry deposition scheme in the Lagrangian dispersion model, NAME are described. Improvements are made for situations where material is not well mixed within the boundary layer and for sedimenting heavy particles. The revised scheme overcomes computational noise, common in Lagrangian dispersion models, with a manageable or no increase to the computational cost. The result is a scheme which is well suited to all situations and which has better interaction between the advection (mean and turbulent), deposition and sedimentation.
\end{abstract}

Key words: dry deposition, elevated sources, entrainment scheme, Lagrangian dispersion model, sedimentation.

\section{INTRODUCTION}

Significant amounts of pollutants are known to be lost from the atmosphere through deposition processes. Consequently. deposition of pollutants has long been recognised as an important component of atmospheric dispersion models. In the absence of precipitation, dry deposition and the sedimentation of heavy particles are key loss processes. This paper describes recent revisions to the dry deposition and sedimentation schemes in the Lagrangian atmospheric dispersion model, NAME (Jones et al., 2007). We describe the issues addressed, the problems encountered within the Lagrangian framework and how these issues were resolved to give more accurate, but efficient, modelling of these loss processes in a range of situations.

\section{DRY DEPOSITION}

Dry deposition in NAME is applied on a particle basis and uses the concept of a deposition velocity, $v_{d}$. The flux of pollutant to the ground is proportional to the concentration of pollutant where the constant of proportionality is given by the deposition velocity. The deposition velocity is determined using a resistance analogy

$$
v_{d}=\frac{1}{R_{a}+R_{b}+R_{c}},
$$

where $R_{a}$ is the aerodynamic resistance representing the efficiency with which material is transported from a reference height to the immediate vicinity of the surface by turbulence, $R_{b}$ is the laminar layer resistance used to specify the resistance to transport by molecular diffusion across the thin quasi-laminar layer adjacent to the surface, and $R_{c}$ is the surface resistance which characterises the efficiency with which pollutant is captured by the surface.

Traditionally, all particles which are within the boundary layer at the end of the model time-step are subject to dry deposition in NAME. This is a common modelling technique used in applied Lagrangian models and, despite the fact that dry deposition is a loss process which occurs at the interface with the surface, is appropriate if the pollutant is well mixed within the boundary layer. In addition, this technique gives the added benefit of reasonably smooth deposition fields rather than suffering from computational noise - a common problem in Lagrangian models. In situations, however, where pollutant is not well mixed within the boundary layer, for example, near to the source, this method does not give an accurate representation of dry deposition. An elevated release within the boundary layer would not, in reality, dry deposit until it was mixed down to the ground at some distance from the source. Under this modelling technique, however, the pollutant would incorrectly be subject to dry deposition at the source. Consequently, the concept of a deposition height, $z_{s}$, was introduced as a parameter which defines the height in the model below which particles are subject to dry deposition and which the user can choose to vary for situations, such as near source problems, where pollutant cannot be assumed to be well mixed within the boundary layer.

\section{Varying the deposition height}

The effect of varying the deposition height over a range from $3 \mathrm{~m}$ to a boundary layer depth of $1000 \mathrm{~m}$ was tested within NAME. The reference height used in the calculation of $R_{a}$ is taken to be half the deposition height. The random walk scheme in NAME calculates turbulent velocity components using either an inhomogeneous velocity memory turbulence scheme, with a small model time-step and turbulence parameters which vary with height, or a homogeneous diffusive scheme (no velocity memory), with a larger time-step and turbulence parameters which are constant with height. Particles are reflected at the ground and, for the homogeneous scheme, an entrainment scheme is used to deal with the discontinuity in the turbulence parameters at the boundary layer top (Thomson et al., 1997). Results, obtained using the inhomogeneous turbulence scheme, are good for a range of deposition heights (Figure 1). For an initial uniform concentration profile within the boundary layer, the amount of material dry deposited is not significantly dependent on the choice of the deposition height and the evolution of the concentration profile seems appropriate. Results are not so good using the homogeneous turbulence scheme. In particular, two unrealistic features in the concentration profiles are evident (see Figure 3). Firstly, there is a step change in the concentration profiles at the deposition height $\left(z_{s}\right)$. Secondly, for small deposition heights it is possible to not deposit enough material (see green profile $\left(z_{s}=3 \mathrm{~m}\right)$ in Figure 3). This under-deposition of material can be explained by the fact that, with a large 
time-step, there is insufficient material below the deposition height to deposit the required amount at the end of the model time-step. Both these problems can probably be resolved by reducing the model time-step but this somewhat defeats the point of an inexpensive homogeneous scheme. We choose, instead, to calculate, for each particle, the fraction, $f$, of the time-step for which the particle is below the deposition height. By using $f$ in the dry deposition scheme, all particles which have been below the deposition height during the model time-step $(f>0)$ contribute to the dry deposition and not just those which are below the deposition height at the end of the time-step. The dry deposition for a particle representing a mass, $m$, of material is given by

$$
\Delta m=m\left[1-\exp \left(-\frac{v_{d}}{z_{s}} f \Delta t\right)\right],
$$

where $\Delta m$ is the mass loss, $V_{d}$ is the deposition velocity and $\Delta t$ is the time-step. The introduction of $f$ removes the unrealistic features and also improves the computational noise issue of the deposition field without an apparent effect on the overall cost of the deposition scheme (see Figure 5). Nonetheless, small values of $z_{s}$ will reduce the number of particles contributing to the dry deposition field and hence the user may need to increase the number of particles in the model run to overcome computational noise. Increasing the number of model particles will clearly have implications on the cost of the model run.

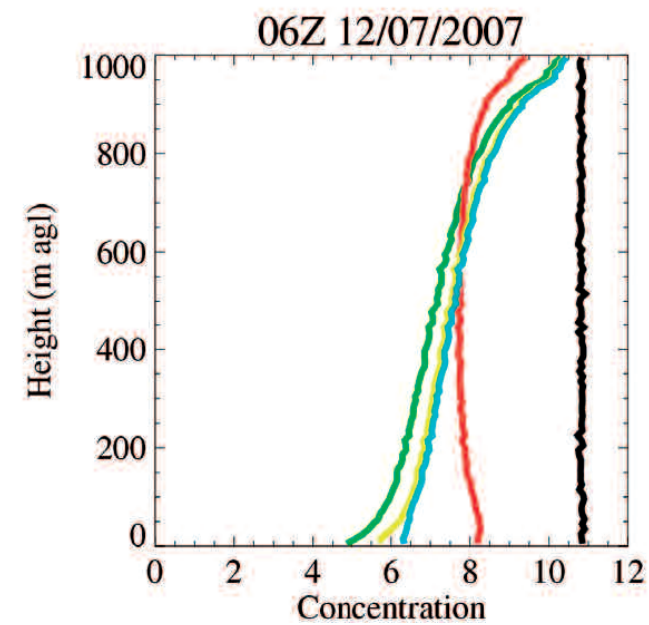

(a) 6 hours

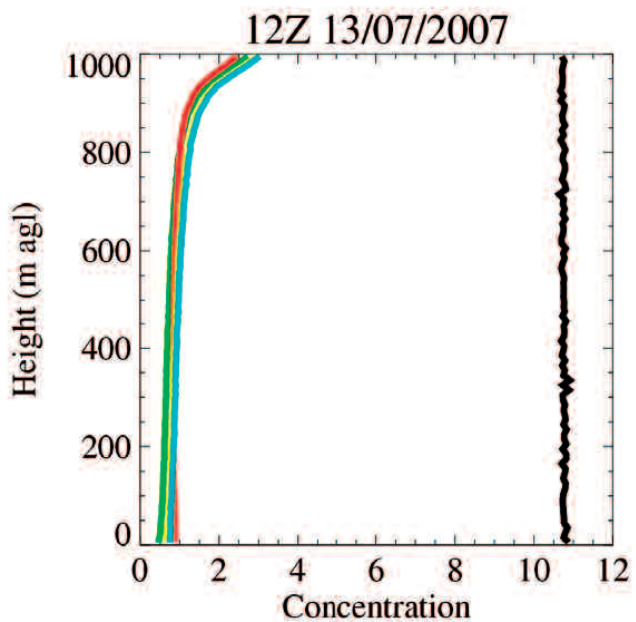

(b) 36 hours

Figure 1. NAME concentration profiles using the inhomogeneous turbulence scheme and varying deposition heights: $1000 \mathrm{~m}$ (red), $100 \mathrm{~m}$ (blue), $30 \mathrm{~m}$ (yellow) and $3 \mathrm{~m}$ (green). The black curve is a non-depositing species shown for comparison and represents the initial uniform concentration.

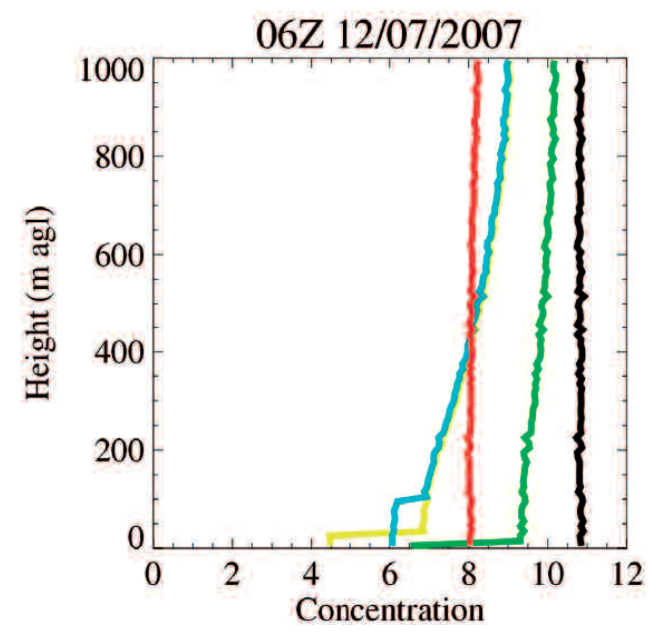

(a) 6 hours

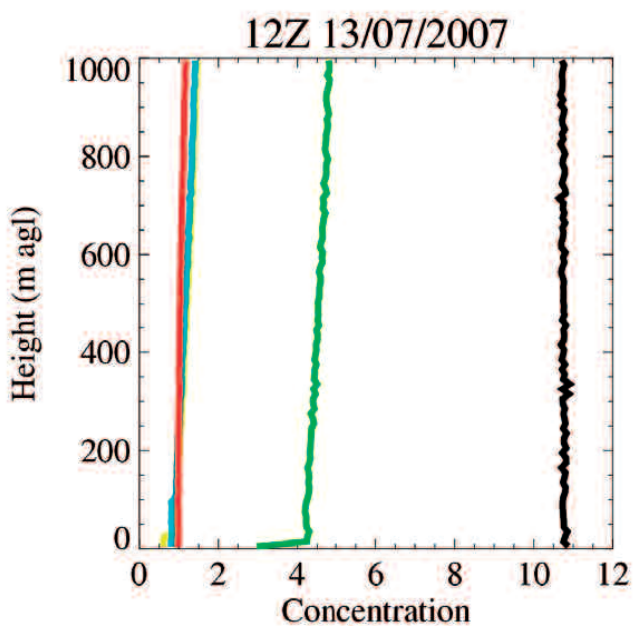

(b) 36 hours

Figure 3. NAME concentration profiles using the homogeneous turbulence scheme and varying deposition heights: $1000 \mathrm{~m}$ (red), $100 \mathrm{~m}$ (blue), $30 \mathrm{~m}$ (yellow) and $3 \mathrm{~m}$ (green). The black curve is a non-depositing species and represents the initial uniform concentration. 


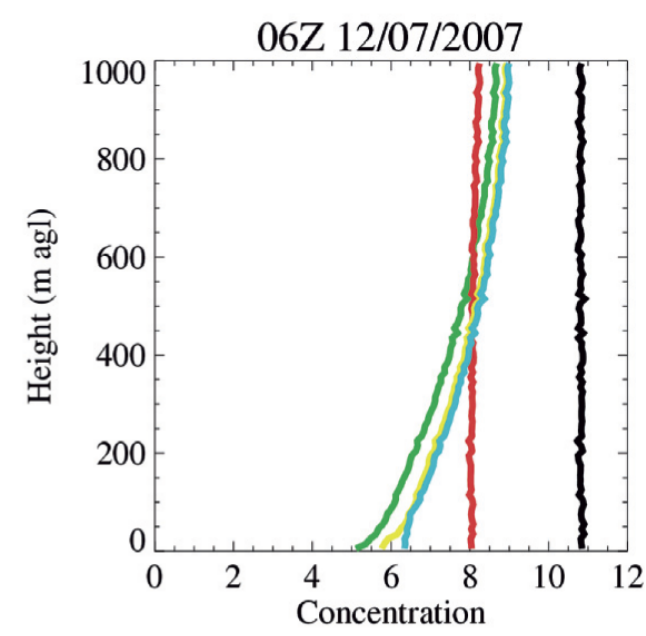

(a) 6 hours

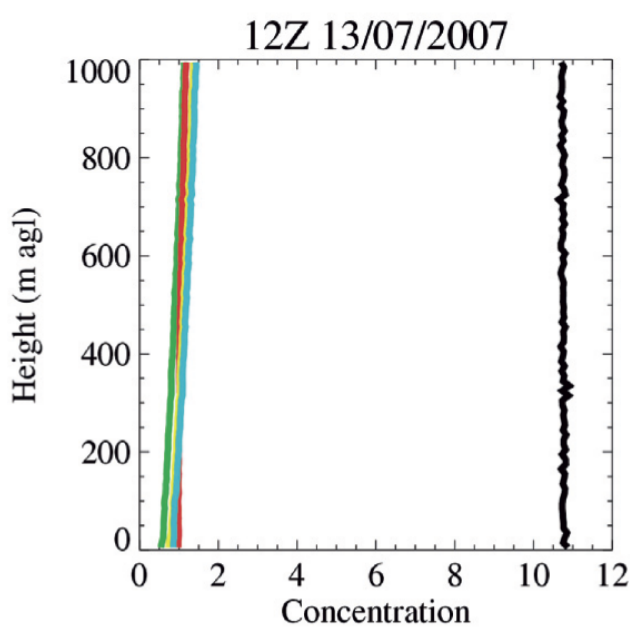

(b) 36 hours

Figure 5. NAME concentration profiles using the homogeneous turbulence scheme and varying deposition heights: $1000 \mathrm{~m}$ (red), $100 \mathrm{~m}$ (blue), $30 \mathrm{~m}$ (yellow) and $3 \mathrm{~m}$ (green). The black curve is a non-depositing species and represents the initial uniform concentration. For each particle the fraction of the time-step below the deposition height is used in the dry deposition calculation.

\section{SEDIMENTATION OF HEAVY PARTICLES}

NAME models gravitational settling of heavy particles, such as volcanic ash, using the concept of a sedimentation velocity, $w_{\text {sed. }}$. The sedimentation velocity is dependent on the particle's diameter and density and a distribution of particle sizes can be represented within NAME with a corresponding distribution of sedimentation velocities. Previously, gravitational settling within NAME was conducted separately from advection by the ambient mean velocity and turbulence, and from dry deposition. Following advection due to the ambient velocity, heavy particles were advected due to sedimentation according to

$$
\frac{d z}{d t}=-w_{\text {sed }} .
$$

Particles which were found to be located below ground following advection due to sedimentation were sedimented out and their mass added to the dry deposition field. For small sedimentation velocities, the proportion of the total number of model particles thereby contributing to the deposition field through sedimentation was small and the resulting deposition field could suffer from computational noise. A decision was therefore taken to incorporate sedimentation into dry deposition via the deposition velocity, $v_{d}$, using

$$
v_{d}=\frac{w_{\text {sed }}}{1-\exp \left(-w_{\text {sed }} / v_{d}^{\prime}\right)},
$$

where $V^{\prime}{ }_{d}$ is the deposition velocity in the absence of sedimentation and is calculated using equation (1) (Underwood, 1999). Equation 4 gives correct limits for $W_{\text {sed }} "\left\langle V^{\prime}{ }_{d}\left(V_{d} \sim V^{\prime}{ }_{d}\right)\right.$ and for $W_{\text {sed }} " V^{\prime}{ }_{d}\left(V_{d} \sim W_{\text {sed }}\right)$ and a better approximation than simple addition of $W_{\text {sed }}$ and $V^{\prime}{ }_{d}$ for the regime in between. Within the advection equation, the sedimentation velocity is damped below $z_{s}$ to compensate. Simultaneous modelling of advection, due to the ambient mean velocity and turbulence, and of sedimentation was implemented to give compatibility with the modification described above, namely the introduction of the fraction of the model time-step, $f$, which requires all components of advection to be treated as a single process. The particle is therefore advected according to

$$
\frac{d z}{d t}= \begin{cases}\overline{w_{a}}+w^{\prime}-w_{\text {sed }}, & z \geq z_{s}, \\ \overline{w_{a}}+w^{\prime}-w_{\text {sed }} \frac{z}{z_{s}}, & z<z_{s},\end{cases}
$$

where $\overline{w_{a}}$ is the mean vertical velocity excluding sedimentation and $w^{\prime}$ is the turbulent vertical velocity component. For the inhomogeneous scheme with velocity memory, the random walk scheme for the evolution of $W^{\prime}$ treats $W_{\text {sed }}$ as part of the mean velocity resulting in a scheme equivalent to that described by Boehm and Aylor (2005). Equation 5 can be easily solved analytically.

\section{Entrainment scheme}

The homogeneous diffusive turbulence scheme, with no velocity memory, has a discontinuity in the turbulence parameters at the boundary layer top and an entrainment scheme is used to determine the fate (reflection or transmission) of model particles impinging on the interface at the boundary layer top. To determine the correct 
entrainment scheme for a non-zero sedimentation velocity we follow the work of Thomson, D. J. et al. (1997) for non-zero mean vertical velocities, with $w_{\text {sed }}$ treated as part of the mean velocity here. In diffusive schemes without velocity memory, the particle turbulent velocities are unphysical and depend on the time-step; however, as discussed in Thomson, D. J. et al. (1997), we can regard them as true velocities for the purpose of designing the entrainment scheme. The flux of particles, with velocity $W$ satisfying $W_{l}<W<W_{2}$, across a surface of constant $z$ is given by

$$
F_{w_{1}}^{w_{2}}(z)=\int_{w_{1}}^{w_{2}} w p_{a}(z, w) d w
$$

where $p_{a}(z, W)$ is the density of particles in $(z, W)$ space. By conservation of flux between two streamlines, we have for a particle incident on an interface from below that the transmission and reflection velocities, $\mathrm{w}_{\mathrm{r}}$ and $\mathrm{w}_{\mathrm{t}}$, respectively, are given by

$$
F_{w_{t}}^{\infty}\left(z_{i_{+}}\right)=F_{w_{i}}^{\infty}\left(z_{i_{-}}\right) \quad \text { and } \quad F_{w_{r}}^{\infty}\left(z_{i_{-}}\right)=F_{w_{i}}^{\infty}\left(z_{i_{-}}\right)
$$

where $w_{i}$ is the incident velocity of the particle (including sedimentation) and $\mathrm{z}_{\mathrm{i}_{+}}$and $\mathrm{z}_{\mathrm{i}_{-}}$are the heights at the top and bottom of the interface, respectively. For a particle incident on the interface from above, the reflection and transmission velocities satisfy

$$
F_{-\infty}^{w_{t}}\left(z_{i_{-}}\right)=F_{-\infty}^{w_{i}}\left(z_{i_{+}}\right) \quad \text { and } \quad F_{-\infty}^{w_{r}}\left(z_{i_{+}}\right)=F_{-\infty}^{w_{i}}\left(z_{i_{+}}\right)
$$

We therefore have that the exit velocity is determined by the incident velocity. The transmission velocity satisfies

$$
\begin{gathered}
-\sigma_{w}(\text { old }) \exp \left[-\frac{\left(w_{i}+w_{\text {sed }}\right)^{2}}{2 \sigma_{w}^{2}(\text { old })}\right]+\sigma_{w}(\text { new }) \exp \left[-\frac{\left(w_{t}+w_{\text {sed }}\right)^{2}}{2 \sigma_{w}^{2}(\text { new })}\right]- \\
\sqrt{\frac{\pi}{2}} w_{\text {sed }}\left[\operatorname{erf}\left(\frac{\mathrm{w}_{\mathrm{i}}+w_{\text {sed }}}{\sqrt{2} \sigma_{w}(\text { old })}\right)-\operatorname{erf}\left(\frac{\mathrm{w}_{\mathrm{t}}+w_{\text {sed }}}{\sqrt{2} \sigma_{w}(\text { new })}\right)\right]=0 .
\end{gathered}
$$

$\sigma^{2}{ }_{\mathrm{w}}$ is an effective velocity variance within the diffusive random walk scheme given by $\sigma_{w}($ old $)=\sqrt{2 K_{z}(\text { old }) / \Delta t}$ and $\sigma_{w}($ new $)=\sqrt{2 K_{z}(\text { new }) / \Delta t}$, where $K_{z}$ is the vertical diffusivity, $\Delta t$ is the timestep, 'old' refers to values on the incident side of the interface and 'new' refers to values on the other side of the interface. Equation (9) is solved numerically for $w_{t}$ using interval bisection. If the sign of $w_{t}$ is the same as the sign of $w_{i}$ then the particle is transmitted. Otherwise the particle is reflected with the reflection velocity, $W_{I}$, found by solving

$$
\begin{gathered}
-\sigma_{w}(\text { old }) \exp \left[-\frac{\left(w_{i}+w_{\text {sed }}\right)^{2}}{2 \sigma_{w}^{2}(\text { old })}\right]+\sigma_{w}(\text { old }) \exp \left[-\frac{\left(w_{r}+w_{\text {sed }}\right)^{2}}{2 \sigma_{w}^{2}(\text { old })}\right]- \\
\sqrt{\frac{\pi}{2}} w_{\text {sed }}\left[\operatorname{erf}\left(\frac{\mathrm{w}_{\mathrm{i}}+w_{\text {sed }}}{\sqrt{2} \sigma_{w}(\text { old })}\right)-\operatorname{erf}\left(\frac{\mathrm{w}_{\mathrm{r}}+w_{\text {sed }}}{\sqrt{2} \sigma_{w}(\text { old })}\right)\right]=0,
\end{gathered}
$$

by interval bisection. For non-sedimenting species $\left(w_{\text {sed }}=0\right)$, equations $(9)$ and (10) can be solved explicitly to give

In this case, if

$$
w_{t}^{2}=\sigma_{w}^{2}(\text { new })\left[\frac{w_{i}^{2}}{\sigma_{w}^{2}(\text { old })}+\log \left(\frac{\sigma_{w}^{2}(\text { new })}{\sigma_{\mathrm{w}}^{2}(\text { old })}\right)\right] \quad \text { and } \quad w_{r}=-w_{i} .
$$

$$
\frac{w_{i}^{2}}{\sigma_{w}^{2}(\text { old })}+\log \left(\frac{\sigma_{w}^{2}(\text { new })}{\sigma_{\mathrm{w}}^{2}(\text { old })}\right)>0
$$

the particle is transmitted with velocity $w_{t}$. Otherwise, the particle is reflected with velocity $W_{r}$ These changes come with an increase of about $15 \%$ in the computational cost for sedimenting species due to solving equations (9) and (10) numerically.

\section{Assessment of model performance}

This change to the modelling procedure and entrainment scheme was tested within NAME using a deposition height and a boundary layer depth of $1000 \mathrm{~m}$. Figure 7 shows the concentration profiles 18 hours after the start of a model run using an initial uniform concentration profile up to a height of $10 \mathrm{~km}$, for an inert tracer (black), a depositing tracer (green) and a depositing and sedimenting species (red) of (a) small sedimenting particles (with a negligible sedimentation velocity) and (b) large sedimenting particles (with a significant sedimentation velocity). The inert tracer maintains a constant concentration profile (shown in black) as expected. The depositing tracer profile (shown in green) is subject to dry deposition below the deposition height, reducing concentrations within the boundary layer over time. Uniform concentrations are, however, maintained within the boundary layer, which is well mixed (in agreement with the red curve in Figure 5). At upper levels, concentrations remain at initial values. Low levels of turbulence above the boundary layer together with entrainment of material into the boundary layer result in a reduction in concentrations from initial values just above the boundary layer top. The concentration profiles for the 
sedimenting species (shown in red) are pleasing. The concentration profile of small sedimenting particles agrees well with the tracer concentration profile. A step change in the concentration profile of large sedimenting particles forms at the interface at the boundary layer top. This is not unrealistic, however, since within the boundary layer material falls under sedimentation whereas turbulent mixing acts to maintain a well mixed constant concentration profile. The resulting concentration profile is therefore a result of both processes. For large sedimenting particles, there is a general decrease in concentrations at upper levels due to the finite upper range of the initial uniform distribution.

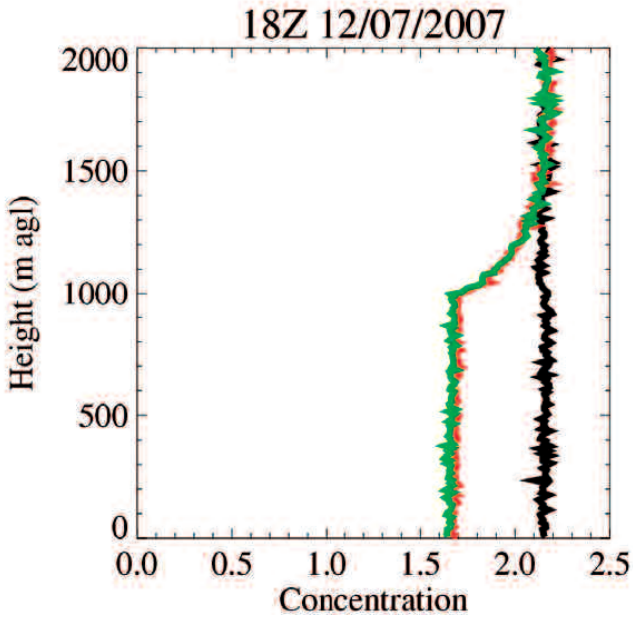

(a) small particles

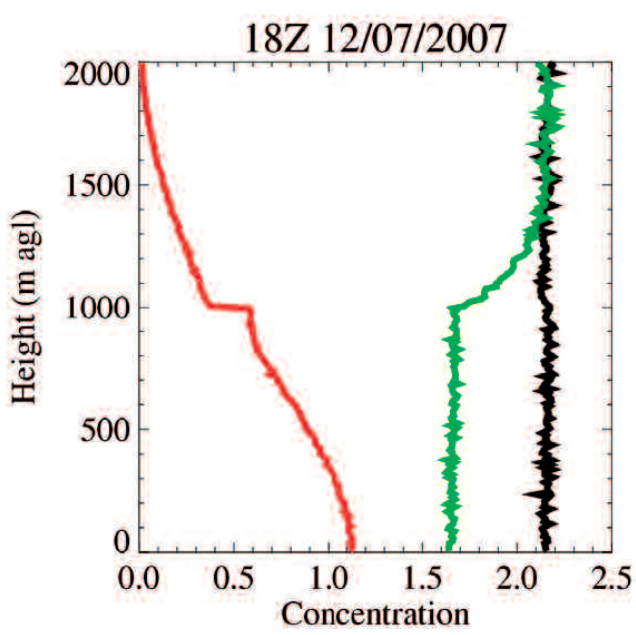

(b) large particles

Figure 7. NAME concentration profiles of an inert tracer (black), a depositing tracer (green) and a depositing and sedimenting species (red) of (a) small particles and (b) large particles, using the homogeneous turbulence scheme and an entrainment parametrization scheme where the exit velocity of the particle at the boundary layer top is determined by its incident velocity.

\section{CONCLUSIONS}

Improvements have been made to the NAME deposition, sedimentation and advection schemes resulting in a better, more complete treatment of these processes which is well behaved in all situations and which does not suffer from computational noise - a common problem in Lagrangian models. The concept of a deposition height has been introduced to allow more accurate modelling of dry deposition in near source problems and in other situations where pollutant is not well mixed in the boundary layer. For small values of the deposition height, the user may need to increase the number of model particles to overcome computational noise, which will, in turn, have implications on the cost of the model run. Changes to the modelling of gravitational settling of heavy particles resulted in sedimentation being incorporated into the main advection and dry deposition routines in NAME. For the homogeneous turbulence scheme, a change to the boundary layer top entrainment parametrization was required, with the exit velocity now determined by the incident velocity. In general, the equation for the exit velocity cannot be solved explicitly and so there is a noticeable, but manageable, increase in the cost due to numerically solving this equation.

\section{REFERENCES}

Boehm, M.T. and D.E. Aylor, 2005: Lagrangian stochastic modelling of heavy particle transport in the convective boundary layer. Atmos. Environ., 39, 4841-4850.

Jones, A., D. Thomson, M. Hort and B. Devenish, 2007: The U.K. Met Office's next-generation atmospheric dispersion model. NAME III, in Air Pollution Modeling and its Application XVII, Borrego, C. and A-L. Norman (Eds.), Springer, 746 pp.

Thomson, D.J, W.L. Physick and R.H. Maryon, 1997: Treatment of interfaces in random walk dispersion models. $J$. Appl. Meteor., 36, 1284-1295.

Underwood, B.Y., 1999: A review of dry and wet deposition. AEAT-5382, AEA Technology plc, UK. 